\title{
A Case of Pyogenic Spondylodiscitis Caused by Campylobacter fetus for Which Early Diagnosis by Magnetic Resonance Imaging Was Difficult
}

\author{
Atsushi Tanaka, Jun Takahashi, Hiroki Hirabayashi, Nobuhide Ogihara, Keijiro Mukaiyama, \\ Masayuki Shimizu, Hiroyuki Hashidate, Hiroyuki Kato
}

Department of Orthopaedic Surgery, Shinshu University School of Medicine, Matsumoto, Japan

\begin{abstract}
The purpose of this case report was to report a rare case of pyogenic spondylodiscitis caused by Campylobacter fetus. A 37 -year-old male presented with fever and low back pain. By lumbar magnetic resonance imaging (MRI), no abnormal finding was observed at the first presentation. However, low back pain was aggravated, and fever did not improve. Thus, lumbar MRI was repeated on the 26 day after the onset of symptoms, showing abnormal signals at vertebrae and disc spaces, and pyogenic spondylitis was diagnosed. The possibility of pyogenic spondylodiscitis should be taken into account if a patient presents with low back pain and fever, and areas of low signal intensity on a T1-weighted MRI should be carefully examined. When initial MRI does not reveal abnormal findings, repeated MRI after one or two weeks or, more favorably, immediate gadolinium enhancement MRI, are important for patients who have persistent low back pain and fever.
\end{abstract}

Key Words: Resonance imaging, Campylobacter fetus

\section{Introduction}

The most common causative organism of pyogenic spondylodiscitis is Staphylococcus aureus, and it is rarely caused by Campylobacter fetus (C. fetus). Magnetic resonance imaging (MRI) is useful in diagnosis of pyogenic spondylodiscitis; however, in the initial stage of symptoms, the specific finding of high signal usually observed on a T2-weighted image is sometimes absent. We investigated the use of MRI in the early diagnosis of pyogenic spondylodiscitis caused by $C$. fetus and its pathology and treatment.

\section{Case Report}

A 37-year-old male who engages in construction work (manual labor) with unremarkable medical history pre- sented at his nearby hospital with fever $\left(\geq 39^{\circ} \mathrm{C},<40^{\circ} \mathrm{C}\right)$ that had no specific trigger nine days before the presentation. The results of a blood test were a white blood cell (WBC) count of $18,900 / \mathrm{mm}^{3}\left(<9,000 / \mathrm{mm}^{3}\right)$ and C-reactive protein (CRP) of $0.62 \mathrm{mg} / \mathrm{dl}(<0.1 \mathrm{mg} / \mathrm{l})$. He was prescribed nonsteroidal anti-inflammatory drugs and oral antibiotics; however, the fever persisted and low back pain had appeared four days before the visit. Body movement became difficult, and he was hospitalized in his nearby hospital. No obvious abnormal finding was observed on lumbar MRI (Fig. 1); however, infection was suspected, and intravenous infusion of cefotiam was started. Low back pain and fever did not improve and lumbar MRI was repeated on the twenty-sixth day after onset (Fig. 2). MRI revealed abnormal signals at vertebrae and disc spaces, and blood culture at the time of hospitalization detected C. fetus. Pyogenic spondylitis was

Received Jun 27, 2011; Revised Jul 29, 2011; Accepted Aug 11, 2011

Corresponding author: Jun Takahashi, MD

Department of Orthpaedic Surgery, Shinshu University School of Medicine,

3-1-1 Asahi, Matsumoto 390-8621, Japan

Tel: +81-263-37-2659, Fax: +81-263-35-8844, E-mail: jtaka@shinshu-u.ac.jp 
diagnosed, and the patient was moved to the internal medicine department of our hospital. Gadolinium enhancement MRI was performed by the request of our internist, and the result strongly suggested pyogenic spondylitis.

He underwent combination therapy of broad spectrum antibiotics; however, the inflammatory reaction did not

T1-WI
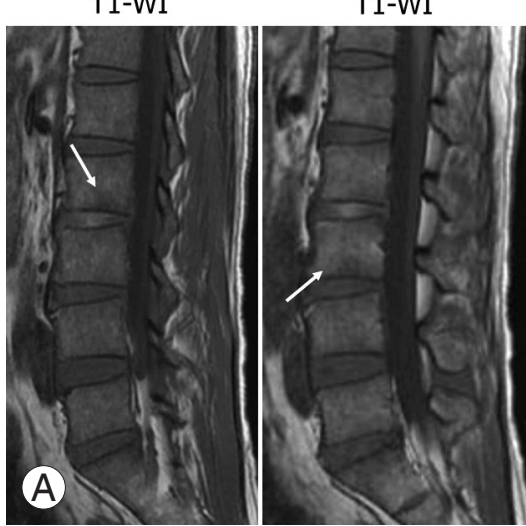

T2-WI

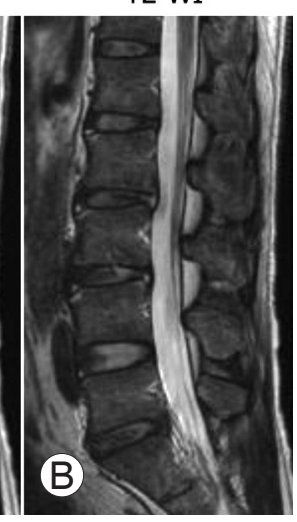

B

Fig. 1. Magnetic resonance imaging at nine days after the onset of symptoms. It was judged that no obviously abnormal findings on both T1-weighted (A) and T2-weighted images (B) were noted at the first diagnosis; however, in retrospective review of the T1-weighted image, a low signal change (arrow) was observed at the L2 and L3 inferior border of the vertebrae.

T1-WI
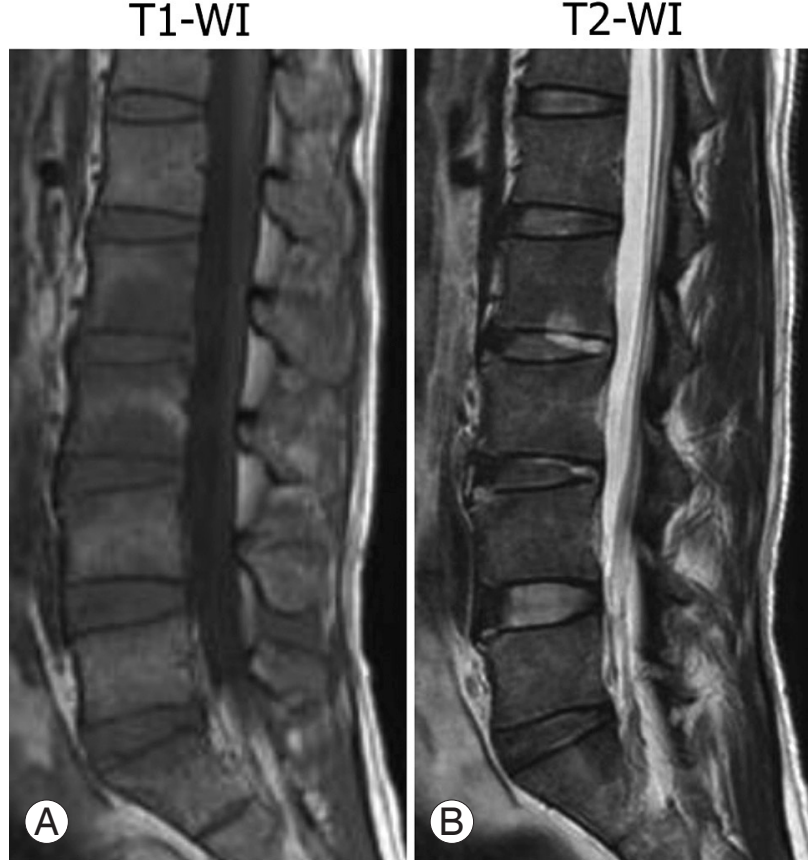

Fig. 2. Magnetic resonance imaging at 26 days after the onset of symptoms. On a T1-weighted image (A), low signal intensity was observed in areas of L2 and L3 vertebrae, and on a T2-weighted image (B), high intensity signal was observed on L2 vertebra and in disc spaces of L2/3 and L3/4. mitigate after six weeks. To clarify if other causative organisms were involved in the inflammation, computed tomography (CT)-guided biopsy was performed after four days' cessation of antibiotics. Biopsy revealed negative results for tuberculosis, atypical mycobacterium, eumycetes, and cultivation of bacteria. He was transferred to our orthopaedic department at 8 weeks, and $C$. fetus was speculated to be the causative organism (Fig. 3). He was treated with intravenous infusion of cefotaxime, ciprofloxacin, and minocycline, and rested with a brace. At 13 weeks after the start of treatment, WBC was in the normal range. At 14 weeks, CRP was negative (Fig. 4), low back pain was improved, and MRI showed reduction of the lesion; thus, the treatment was changed to oral ciprofloxacin and minocycline and the patient discharged. He received outpatient antibiotics, choosing one or two drugs out of ciprofloxacin, minocycline, and erythromycin for 11 months. CRP temporally became negative after 14 weeks of onset, then CRP increased and became negative repeatedly. Finally, CRP was completely negative after 15 months of onset. Lesions had almost disappeared on MRI after one year, and the vertebral body showed high signal intensities on both T1and T2-weighted images (Fig. 5). Activities of daily living were permitted without restriction after the discharge. Light

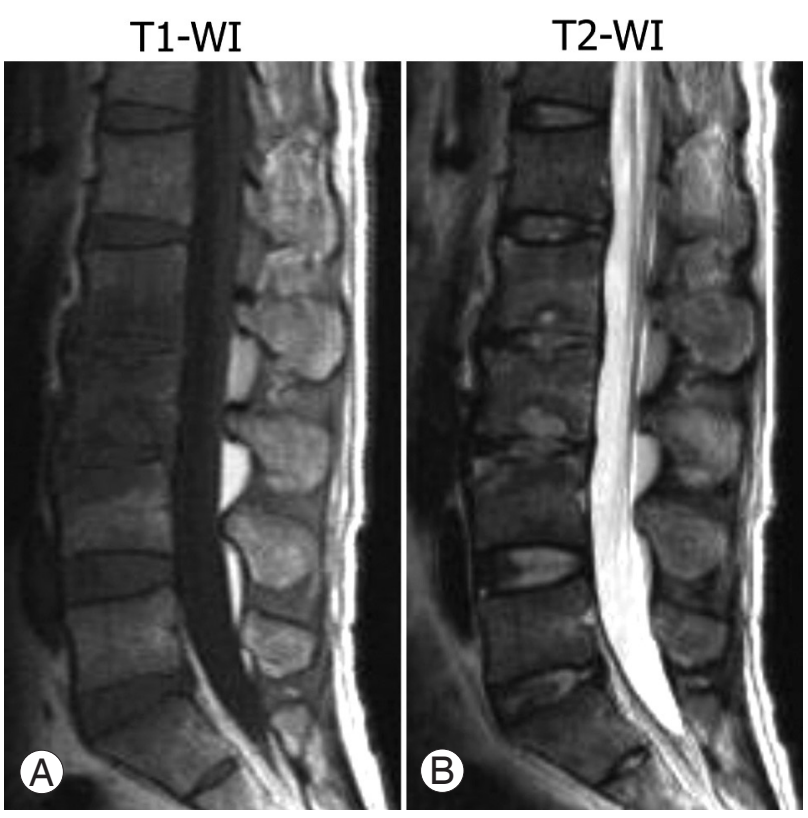

Fig. 3. Magnetic resonance imaging at nine weeks after the start of symptoms. On a T1-weighted image (A), low signal intensity was observed in areas of L2 and L3 vertebrae; on a T2-weighted image (B), a high signal intensity region was observed. Lesions were becoming clearer. 
work and general work was permitted after 6 and 9 months, respectively. After 13 months, back pain had almost disappeared and he performed hard labor without problems.

\section{Discussion}

The sources of $C$. fetus infection include intake of contaminated meat or milk and contact with infected animals, and its onset is frequently observed in immunodeficiency patients including those with diabetes, human immunodeficiency virus infection, or malignant tumor [1-3]. Such infection results in various clinical symptoms and causes various conditions, including infective endocarditis, thrombophlebitis, meningitis, meningoencephalitis, enteritis, and bacteremia, and can result in miscarriage [4]. However, the bone and joint infection rate is as low as $5 \%$ or less, and the majority of bone and joint infections occurs at the hip or knee joint [1]. As a result of our search, only 2 reports of pyogenic spondylitis caused by $C$. fetus were found $[1,5]$. The patient in the present report had no obvious contact with an infection source, and the detailed systemic work-up revealed no disease that causes immunosuppression or any infectious sites other than the spine.

Sensitivity of $C$. fetus to antibiotics had been studied, but optimal antibiotic treatment has not yet been established [6]. Combination therapy with doxycycline and erythromycin had been reported $[1,5]$; however, treatment with erythromycin is not recommended in one report due to the existence of an erythromycin-resistant strain [6]. It is important to select antibiotics considering sensitivity and tissue penetration [6]. We performed intravenous infusion of cefotaxime, ciprofloxacin, and minocycline until the inflammation reaction became negative, and then orally administered 1 or 2 drugs among the three (cycling treatment). In previous reports, oral antibiotic administration was continued 3 to 5 months after the completion of intravenous administration

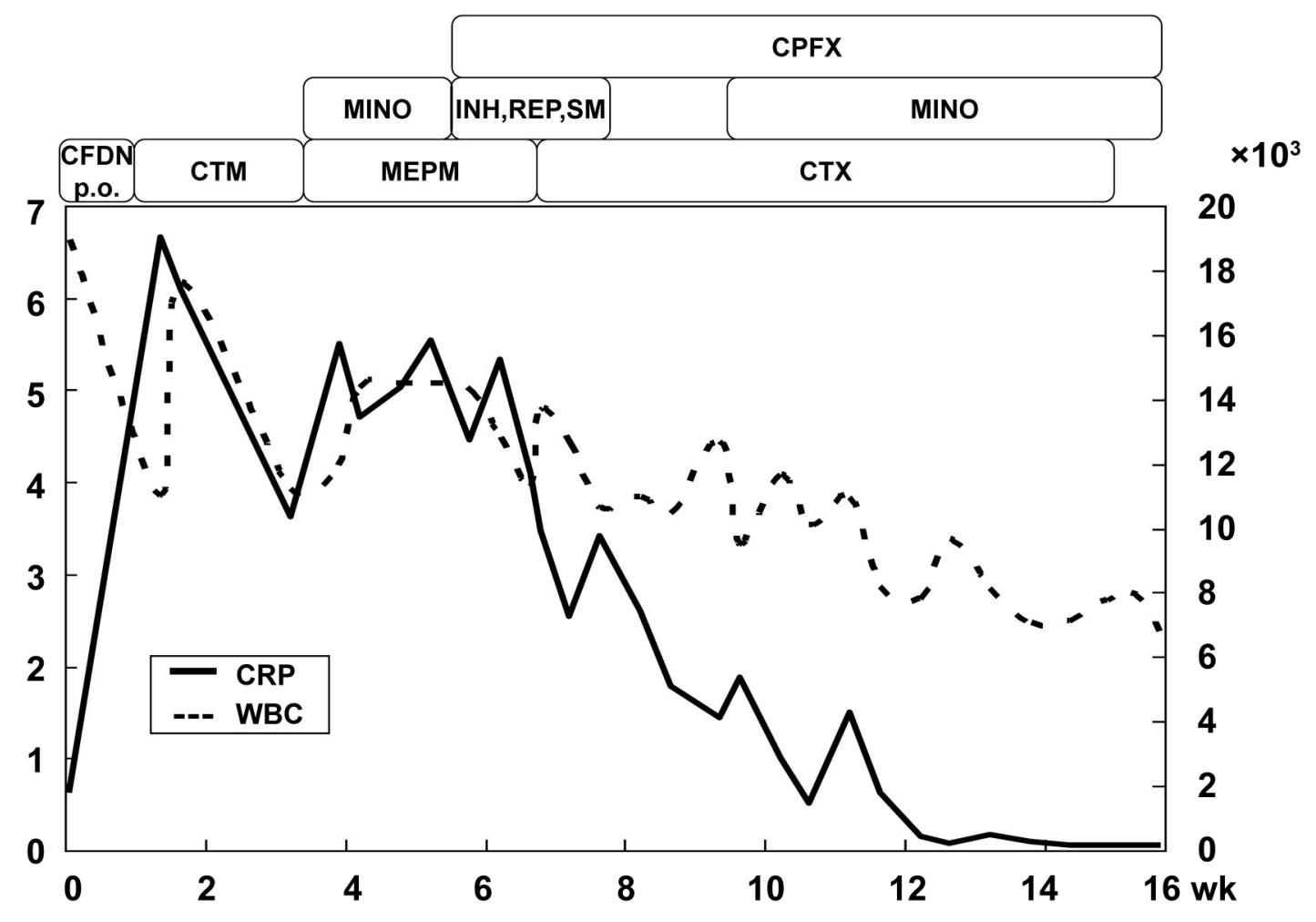

Fig. 4. Cause of treatment during hospitalization. Solid line (left vertical axis) represents CRP $(<0.1)$, and dotted line (right vertical axis) represents WBC $(<9,000)$. Horizontal axis is the time line from the onset of symptoms; the antibiotics used are shown in the upper region of the graph. The patient was moved to our division after eight weeks. He was then intravenously infused with multiple antibiotics and became CRP negative after 14 weeks. WBC: White blood cell, CRP: C-reactive protein, CFDN p.o.: Cefdinir per os, CTM: Cefotiam, MINO: Minocycline, MEPM: Meropenem, INH: Isoniazid, REP: Rifampicin, SM: Streptpmycin, CPFX: Ciprofloxacin, CTX: Cefotaxime. 
$[1,4]$. It is best to comprehensively consider all pertinent clinical and inflammation symptoms and improvement of image findings before determining whether oral antibiotics should be continued.

Some cases of pyogenic spondylitis do not present remarkable clinical findings and frequently result in delayed diagnosis. MRI is the most effective tool in early diagnosis of pyogenic spondylitis, with sensitivity and specificity as high as $96 \%$ and $92 \%$ to $94 \%$, respectively [7]. In the acute phase, T2-weighted images present high signal intensity in the peripheral region of disc and end plate and T1-weighted images present low signal intensity in the large area of inflammation or edema around the seat of the disease [8]. However, cases without abnormal finding by MRI after 2 weeks of the onset of symptoms [9] were reported, and cautious diagnosis is required. In the case of the present report, obvious abnormal findings were not found 13 days after onset; however, pyogenic spondylitis had been suspected by clinical findings and blood biochemistry. Because the low back pain had not improved, MRI was repeated 26 days after onset. Areas of low and high signal intensity were found in the vertebral body and disc on T1- and T2-weighted images, respectively, and subsequently, pyogenic spondylitis was diagnosed. In reports of MRI images of pyogenic spondylitis, the vertebral body first presented a low signal

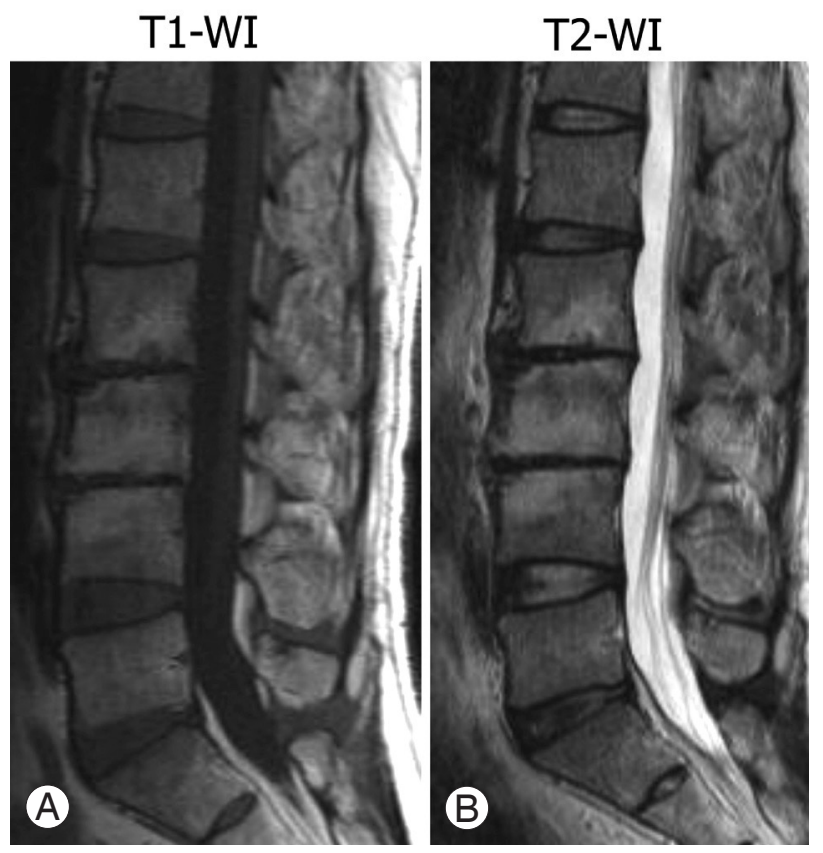

Fig. 5. Magnetic resonance imaging at one year after the onset of symptoms. The lesion had disappeared, and L2 and L3 vertebrae showed high signal intensity on both a T1weighted image (A) and T2-weighted image (B). intensity on a T1-weighted image, then a high signal intensity was observed on a T2-weighted image [10,11]. Likewise, retrospective evaluation of our case revealed that the first MRI showed slight low signal intensity of the vertebral body on a T1-weighted image (Fig. 1). However, in some cases, an MRI image within 2 weeks after onset does not reveal obvious abnormal findings, and thus repeated MRI is necessary if persistent fever or low back pain is observed. In the repeated MRI after the early MRI with no abnormal findings, careful evaluation of the T1-weighted image is important.

In addition, MRI is useful for the evaluation of disease progression and judgment of the treatment. Along with the resolution of symptoms, the low signal intensity areas on a T1-weighted image and the high signal intensity on a T2weighted image show lesions decrease and disappear [8]. During remission, the high signal intensity sometimes appears on a T1-weighted image of the vertebral body, and it is considered to show the regeneration of fatty bone marrow [12]. In our case, lesions disappeared after 1 year, and high signal intensity was observed on a T1-weighted image.

In conclusion, we reported pyogenic spondylitis caused by $C$. fetus, which is rarely observed. In the diagnosis of patients who present with low back pain and fever, it is important to consider pyogenic spondylitis and to look for low signal intensity regions on T1-weighted MRI images. When the initial MRI does not reveal abnormal findings, repeated MRI after one or two weeks or, more favorably, immediate gadolinium enhancement MRI, are important for patients who have persistent low back pain and fever.

\section{REFERENECES}

1. Mathieu E, Koeger AC, Rozenberg S, Bourgeois P. Campylobacter spondylodiscitis and deficiency of cellular immunity. J Rheumatol 1991;18:1929-31.

2. Allerberger F, Kasten MJ, Anhalt JP. Campylobacter fetus subspecies fetus infection. Klin Wochenschr 1991;69:813-6.

3. Bracikowski JP, Hess IE, Rein MF. Campylobacter osteomyelitis. South Med J 1984;77:1611-3.

4. Schmidt U, Chmel H, Kaminski Z, Sen P. The clinical spectrum of Campylobacter fetus infections: report of five cases and review of the literature. Q J Med 1980;49:431-42.

5. Yamashita K, Aoki Y, Hiroshima K. Pyogenic vertebral osteomyelitis caused by Campylobacter fetus 
subspecies fetus. A case report. Spine (Phila Pa 1976) 1999;24:582-4.

6. Tremblay C, Gaudreau C, Lorange M. Epidemiology and antimicrobial susceptibilities of 111 Campylobacter fetus subsp. fetus strains isolated in Quebec, Canada, from 1983 to 2000. J Clin Microbiol 2003;41:463-6.

7. Butler JS, Shelly MJ, Timlin M, Powderly WG, O'Byrne JM. Nontuberculous pyogenic spinal infection in adults: a 12-year experience from a tertiary referral center. Spine (Phila Pa 1976) 2006;31:2695-700.

8. Akagi S. Diagnosis of spinal infections in early stage. Orthop Surg Traumatol 2009;52:11-9
9. Yoshikawa T, Maeda M, Ueda Y, et al. Magnetic resonance imaging in the early phase of pyogenic spondylitis: a report of four cases. J Orthop Sci 1997;2:16-23.

10. Fischer U, Vosshenrich R. Osteomyelitis (spinal infection, spondylitis, spondylodiscitis) of the dens axis. Rontgenblatter 1990;43:54-7.

11. Kramer J, Stiglbauer R, Wimberger D, Imhof H. MRI of spondylitis. Bildgebung 1992;59:147-51.

12. Smith AS, Weinstein MA, Mizushima A, et al. MR imaging characteristics of tuberculous spondylitis vs vertebral osteomyelitis. AJR Am J Roentgenol 1989;153:399-405. 